\title{
Influence of Heat Treatment on Defect Structures in Single-Crystalline Blade Roots Studied by X-ray Topography and Positron Annihilation Lifetime Spectroscopy
}

\author{
JACEK KRAWCZYK, WŁODZIMIERZ BOGDANOWICZ, \\ ANETA HANC-KUCZKOWSKA, ANNA TONDOS, and JAN SIENIAWSKI
}

\begin{abstract}
Single-crystalline superalloy CMSX-4 is studied in the as-cast state and after heat treatment, with material being taken from turbine blade castings. The effect of the heat treatment on the defect structure of the root area near the selector/root connection is emphasized. Multiscale analysis is performed to correlate results obtained by X-ray topography and positron annihilation lifetime spectroscopy (PALS). Electron microscopy observations were also carried out to characterize the inhomogeneity in dendritic structure. The X-ray topography was used to compare defects of the misorientation nature, occurring in as-cast and treated states. The type and concentration of defects before and after heat treatment in different root areas were determined using the PALS method, which enables voids, mono-vacancies, and dislocations to be taken into account. In this way, differences in the concentration of defects caused by heat treatment are rationalized.
\end{abstract}

https://doi.org/10.1007/s11661-018-4704-2

(C) The Author(s) 2018

\section{INTRODUCTION}

THE single-crystalline, Ni-based superalloys are widely used for production of high-pressure and high-temperature turbine components in aerospace and energy industry sectors. Due to the extreme work conditions of blades, especially high mechanical and thermal stresses, the specific properties with low concentration of structural defects are needed..$^{[1-3]}$ Nowadays, the CMSX-4 single-crystalline superalloy is commonly used by industry; castings produced in this way are suitable candidates for defect characterization studies.

Directional dendritic solidification by the Bridgman technique is widely used for production of single-crystalline blades made from superalloys. Production technology and the complex shape of blade castings produce dendritic arrays that allow for the possibility of many defects to be produced during solidification. The defects

JACEK KRAWCZYK, WŁODZIMIERZ BOGDANOWICZ, ANETA HANC-KUCZKOWSKA, and ANNA TONDOS are with the Institute of Materials Science, University of Silesia in Katowice, 41-500 Chorzow, Poland. Contact e-mail: jacek.krawczyk@us.edu.pl JAN SIENIAWSKI is with the Department of Materials Science, Rzeszów University of Technology, 35-959 Rzeszow, Poland.

Manuscript submitted March 14, 2018.

Article published online June 1, 2018 may be related to the inhomogeneity of the chemical composition, morphology and size of $\gamma^{\prime}$ particles, and the inhomogeneity of crystal orientation. Therefore, blades are subjected to complex heat treatment after casting, among others, to eliminate the chemical heterogeneity caused by directional dendritic crystallization. ${ }^{[4]}$ The heat treatment parameters are appropriately selected to create new $\gamma / \gamma^{\prime}$ array (reprecipitation ${ }^{[4]}$ ) with optimized properties, caused by more homogeneous morphology, size, and chemical composition. Some defects of macro-, micro-, and nano-scale are recreated in the treated blades, but some of them may be inherited from the as-cast state. All of them to some degree or other will influence the mechanical properties of blades and may cause damage during operation. ${ }^{[5-9]}$ The macroscopic inhomogeneity in crystal orientation (e.g., subgrain boundaries or misoriented bands of dendrites, bended during crystallization) may be one of the inherited defects. These defects are generally related to the crystallization of complex shape casts. ${ }^{[4,10-12]}$

The inhomogeneity of crystal orientation may be characterized by X-ray diffraction topography in reflective geometry. ${ }^{[13,14]}$ This imaging technique - which represents two-dimensional mapping of diffracted $\mathrm{X}$-rays local intensity - is capable of providing information on the distribution and nature of structural defects in single-crystalline materials. The technique is sensitive to changes in local orientations of diffraction (atomic) planes and their spacing. ${ }^{[15,16]}$ The method allows the 
visualization of the differences in crystal orientation (misorientation) of neighboring areas accurate to arc minutes. However, the analyzed areas must have a size of $1 \mathrm{~mm}^{2}$ or higher. The boundaries between such areas (macroscopic low-angle boundaries, LABs) are created by microscopic defects like dislocations, and additionally, they may be related to the vacancies. This means that the general description of defect structure of blades must refer to defects over a wide spatial size ranging from nanometer up to millimeter scale, which are mutually related to each other. Determining the relationship of different-scale defects, which is the basis of the multiscale analysis, helps to determine the reasons for their creation.

Additionally, the macroscopic inhomogeneity of crystal orientation may be related to sub-micrometer-scale defects, which are difficult or impossible to reveal by SEM or TEM methods due to their small analyzed area. The positron annihilation lifetime spectroscopy (PALS), using a macroscopic area, may enable it. PALS is a promising nondestructive method of quality control of technologically important materials employed in various fields of science and technology. Positrons interact for example with foreign atoms, points, or linear defects of structure. Positrons are trapped preferentially in atomic defects leading to an extended positron lifetime. The advantage of this method is the detection of small concentrations of defects that could not be detected by other methods. ${ }^{[17]}$ Positron techniques have been used for studies of defect behavior in the $\mathrm{Ni}_{3} \mathrm{Al}$ system ${ }^{[18]}$ and polycrystalline Ni-based superalloys, ${ }^{[19]}$ but there are no results as yet for investigation of defects inhomogeneity in the single-crystalline turbine blades.

It is emphasized that the structural defects are mainly created in the so-called critical areas of the blade. The single-crystalline blades possess critical areas with regard to the crystallization process (selector-root and root-airfoil connection areas, thin-walled areas) and to the operation loads (areas near the root-airfoil connection, thin-walled areas of trailing edge, tip area). ${ }^{[20,21]}$ Regarding to the growth structure of dendrites set, the most affected area is situated in the root part of the blade, near the connection with the selector, where the step-change geometry of the castings occurs. ${ }^{[22]}$ The above justifies the selection of these regions for attention in this article. Its overarching aim of the study is to analyze and compare the defects structure of CMSX-4 single-crystalline blade root areas, located near the selector-root connection, in an as-cast state and after heat treatment using X-ray topography and the PALS method.

\section{EXPERIMENTAL}

The investigated roots of single-crystalline turbine blades, made of CMSX-4 superalloy, were obtained by directional crystallization via Bridgman technique at a withdrawal rate of $5 \mathrm{~mm} / \mathrm{min}$. with the use of the spiral selector with [001] orientation (Figure 1). The blade roots were cut into two parts: $\mathrm{C}$ and $\mathrm{T}$, perpendicular to the withdrawal direction $G$ (Figure 1). The cutting plane
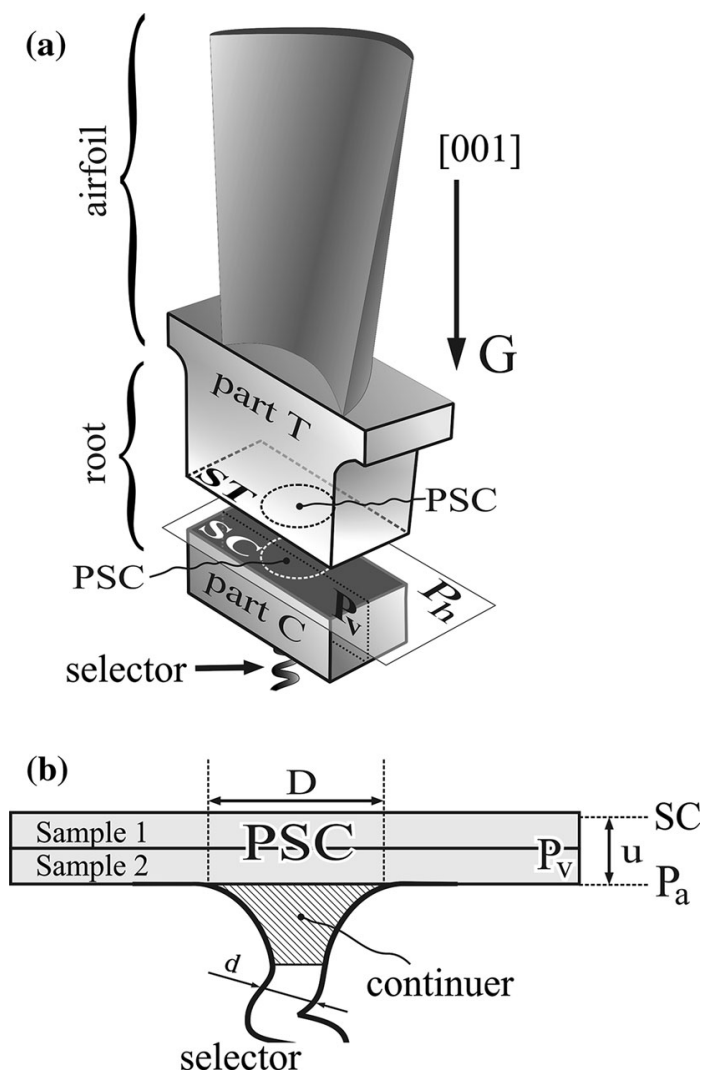

Fig. 1-(a) Illustration of blade parts and (b) samples for PALS preparation; $P_{\mathrm{v}}-$ section of part $\mathrm{C}$.

$P_{\mathrm{h}}$ (Figure 1) was located near the surface $P_{\mathrm{a}}$ (Figure 1(b)) of the selector-root connection $(u=3.5 \mathrm{~mm})$. The $\mathrm{ST}$ surface was a mirror-reflection image of the SC surface. The SC surface of C-parts (Figure 1(a)) was studied in the as-cast state, and the ST surface was analyzed after heat treatment of T-parts. Section $P_{\mathrm{v}}$ in Figure 1(b) reveals the geometry of the selector-root connection with the use of a selector continuer higher in diameter on the plane $P_{\mathrm{a}}$ in relation to the diameter of selector channel $(D>d)$. The PSC in Figure 1 is the projection of a selector continuer on all transverse sections of the root.

The heat treatment of part $\mathrm{T}$ was performed by several steps, consisting of convection heating to $950{ }^{\circ} \mathrm{C}$ (in a helium-protective atmosphere), radiation heating to $1350{ }^{\circ} \mathrm{C}$ (in vacuum), solution annealing, and finally aging. The temperature-time settings for annealing were: $1277^{\circ} \mathrm{C} / 4 \mathrm{~h} \rightarrow 1287{ }^{\circ} \mathrm{C} / 2 \mathrm{~h} \rightarrow 1296{ }^{\circ} \mathrm{C} / 3 \mathrm{~h} \rightarrow 1304{ }^{\circ} \mathrm{C} /$ $3 \mathrm{~h} \rightarrow 1313{ }^{\circ} \mathrm{C} / 2 \mathrm{~h} \rightarrow 1316^{\circ} \mathrm{C} / 5 \mathrm{~h} \rightarrow$ gas furnace quench, and for aging: $1140{ }^{\circ} \mathrm{C} / 6 \mathrm{~h}$ (step 1) and $871{ }^{\circ} \mathrm{C} /$ $20 \mathrm{~h}$ (step 2). The blade production and heat treatment process were performed in the Research and Development Laboratory for Aerospace Materials, Rzeszów University of Technology, Poland, using an industrial ALD Vacuum Technologies furnace.

The metallographic sections of SC and ST surfaces were studied by scanning electron microscopy (SEM), the X-ray diffraction topography, and Laue diffraction. A JEOL JSM-6480 microscope was applied for SEM observations using a backscattered electron (BSE) 
technique. A divergent beam of characteristic $\mathrm{Cu}_{\mathrm{K} \alpha}$ radiation, generated by a microfocus $\mathrm{X}$-ray tube of the PANalytical system, was used for X-ray topography studies. The topograms were recorded on the AGFA Structurix D7 X-ray film in reflective geometry. The divergent beam of the characteristic radiation coming from a quasi-point $\left(40 \times 40 \mu \mathrm{m}^{2}\right) \mathrm{X}$-ray source illuminates the whole surface of the SC or ST surfaces. ${ }^{[13]}$ The sample (part $\mathrm{C}$ or $\mathrm{T}$ ) coupled with the film oscillates about the axis located on the studied surface. The individual parts of the tested surface, meeting the Bragg condition, are successively recorded on the film. After over a dozen minutes of exposure, the diffraction image (topogram) of the whole analyzed surface was obtained on the film. If for areas close to surface SC of part C or ST for part T, the crystal lattices are rotated relative to each other, then their diffraction images will be mutually shifted in the topogram, creating different types of contrast. Similar displacement in the topograms was created by changes of d-spacing. Specific contrast is also created for other defects that occurred in the single-crystalline materials. ${ }^{[13,23]}$ The misorientation angle may be calculated using the shift value in the topograms. ${ }^{[13]}$ The minimal misorientation angle, determined by X-ray topography method, is on the order of arc minutes, which are much lower than for the EBSD method.

Additionally, the PALS technique was applied to the study defect structure, revealed by X-ray topography on the surfaces SC and ST. PALS relies on the propensity of positrons to become localized at open-volume regions of a solid and the emission of annihilation gamma rays that escape the test system without any final state interaction. The scheme used for sample sandwich packing in the PALS measurements and scheme of positron trapping are illustrated in Figure 2. The sources of positrons are generated by a pair of gamma rays that hold information about the electronic environment around the annihilation site. PALS measures the elapsed time between the implantation of the positron into the material and the emission of annihilation radiation. Positrons are trapped preferentially in atomic defects that in turn have a locally smaller electron density leading to an extended positron lifetime. The PALS technique therefore is a sensitive method to derive sizes and concentration of vacancy-type defects like nano-cavities. The characteristics of annihilation trapping positron are different for various configurations defects. Knowledge of the location of positrons in defect of lattice gives the possibility of the concentration of defects from proportion of trapping positron to free. Here, it was applied in determining the energy of vacancy formation.

Two samples in a sandwich arrangement (Figures 2(a) and 1(b)) for PALS measurements were prepared separately from each C-part and each T-part of the blades. The PALS measurements were performed in two areas (A1, A2 of Figure 2(b)) of each sandwich at room temperature with a conventional fast-fast spectrometer of 270-ps time resolution. The area A1 relates to the PSC area and A2 to the area outside it. The positron source with the activity of about $370 \mathrm{kBq}$, covered with a $5-\mu \mathrm{m}$ Ni foil, was used. For each sandwich, a series of (a)

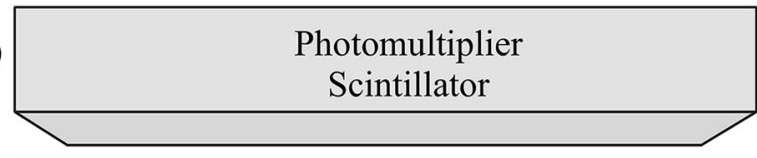

Sample 1

$\longrightarrow \mathrm{Al}_{\mathrm{A} 2}$

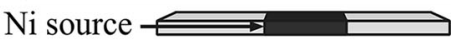

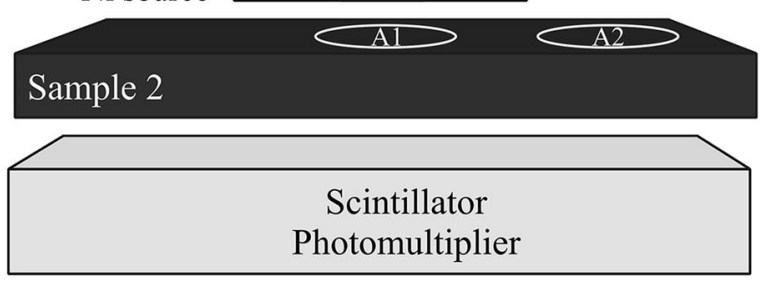

(b)

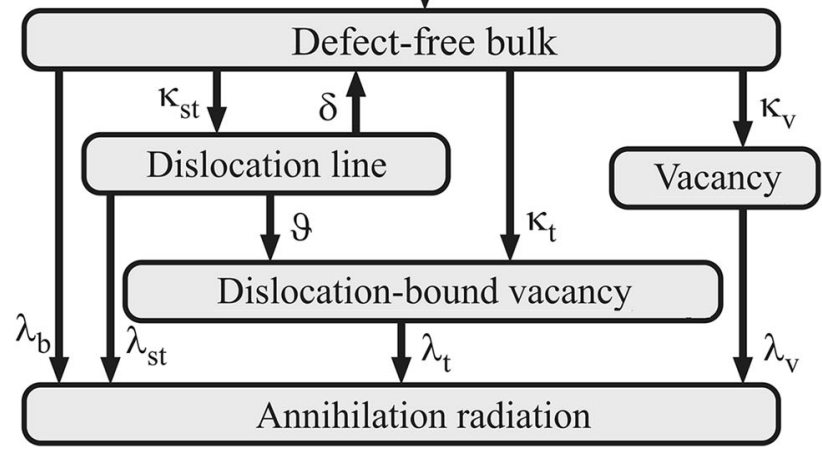

Fig. 2-(a) Scheme of samples sandwich arrangement in PALS measurements, (b) scheme of the positron annihilation processes according to the three-state-trapping model: positron trapping in complex defects: positron trapping in a vacancy and dislocation.

spectra was recorded. Then, the spectra were added together by means of a special procedure accounting for the drift of the zero-time channel. In this way, a resultant spectrum of very high statistics (at least 107 counts) was obtained. The data were analyzed using a least-square fitting procedure, ${ }^{[24]}$ which, contrary to the other existing programs, enables fitting not only a single spectrum but also a series of spectra. The simultaneous fitting leads to reduction of the number of the free fitting parameters because some of the parameters can have common values for all of the spectra of the series. In the calculations, all the measured spectra were fitted simultaneously with a model, directly implemented within the software code. ${ }^{[24]}$ All spectra were analyzed using the three-state trapping model ${ }^{[24]}$ describing the positron annihilations in the bulk material and two types of defects (Figure 2(b)). The three-state trapping model was described by three parameters, i.e., the positron lifetime in bulk material $\tau_{\mathrm{b}}$, the positron lifetime trapped by defects $\tau$, and the positron trapping rate $\kappa$.

In Figure 2(b), the horizontal bars represent the free state of positrons, i.e., the delocalized state of each positron in bulk material and its localized states (dislocations and vacancy) in two types of defects. $\lambda_{\mathrm{b}}$ is the annihilation rate of the delocalized (free) positron 
from the bulk, $\lambda_{\mathrm{st}}$ and $\lambda_{\mathrm{t}}$ and $\lambda_{\mathrm{v}}$ are the annihilation rates from different type of defects, respectively. $\kappa_{\mathrm{v}}, \kappa_{\mathrm{t}}$, and $\kappa_{\mathrm{st}}$ are the trapping rates into these defects, which are proportional to the defect concentrations. $\vartheta$ are the trapping rate into dislocation-bound vacancy. The term $\delta$ is the detrapping (escape) rate.

All the measured spectra were fitted simultaneously at each parameter relating to the resolution or the contribution of source. This means that such a parameter was constrained to have the same value for each spectrum of the series of spectra analyzed together. The positron lifetimes relating to positron annihilation in the source were fixed. The "source" lifetimes were determined with help of a Si lifetime spectrum of very high statistics. The source contribution of 36.7 pct consisted of three components with lifetimes 125 ps, 386 ps, and $1.97 \mathrm{~ns}$ in the proportion 94.6:4.7:0.7, respectively.

\section{RESULTS}

Metallographic observations of SC surfaces of C-type parts revealed a dendritic structure (Figure 3(a)) typical of the as-cast state. The macro-SEM images, created by merging multiple separate SEM images, allow us to observe the whole section and compare the morphology and distribution of dendrites in different areas of the SC surface. A computer processing was applied for better visibility of dendrites arms direction and type of arrangement (Figure 3(b)). The processing combines creation of skeletal images and modification of contrast. The procedure gives results possessing certain common features with the skeletonization procedure referred to in Reference 25. Skeletonization is a technique whereby a binary image of dendrites is eroded step by step away until the skeleton of the image is obtained. The skeletal image is created as a thin line equidistant from the original edges of the binary dendrites shape. ${ }^{[25]}$ Analyzing images from Figure 3, two different types of dendrite arms arrangement can be distinguished: short-range alternate (inside the center of PSC) and long-range continuous ( $L_{\mathrm{R}}$-type). The center part of the sections consists of dendrites with a homogenous, statistical arrangement. A small disorder in dendrite arm directing (shifts of linear fragments) is noticeable around the PSC area (Figure 3). The mutual arrangement of short-range and long-range arms (depending on the $m$ and $n$ directions) allows visualization of a ring that overlaps the edge of the PSC area and is marked by $R_{\mathrm{C}}$ (Figure 3(b)). The diameter of the ring is equal to the diameter $D$ of the PSC area (Figure 1(b)). The $R_{\mathrm{c}}$ ring may be better visible when the figure is reduced (Figure 3(c)). The largest difference in dendrites morphology occurs in narrow corner areas $\left(K_{1}, K_{2}\right)$. The dendrite arms are elongated along the $n$ direction and fine ternary arms appear. No clear dendritic structure was observed on the metallographic sections of the ST surface of T-type parts. By Laue diffraction, it was stated that the $n$ and $m$ directions correspond to the [010] and [100] crystallographic directions.
Figure 4 presents typical topograms obtained for the SC surface of C-type part (Figure 4(a)) and for the ST surface of T-type part (Figure 4(b)). The ST surface was a mirror-reflection image of the SC surface; therefore, the contrast details in the topograms for the same corresponding locations on the analyzed surfaces (Figures 4(a) and (b)) are mirror-reflected, e.g., the $L_{1 \mathrm{C}}$ contrast band (Figure $4(\mathrm{a})$ ) relate to the $L_{1 \mathrm{~T}}$ band (Figure 4(b)). Additionally, the contrast inversion is visible. It can be observed during a hypothetical move from the corner $K_{\mathrm{C}}$ (Figure $4(\mathrm{a})$ ) to the center of the A2 area: In Figure 4(a), the dark-contrast band is visible first, and in Figure 4(b), the bright-contrast band is visible first.

In both topograms, the R-rings $\left(R_{\mathrm{C}}, R_{\mathrm{T}}\right)$ of contrast with change in intensity of the rim of the PSC are visible. The $R_{\mathrm{C}}$ ring (Figure 4(a)) consists of two sub-rings: the external one of $S_{\mathrm{RC} 1}$ width with higher contrast intensity (Figure $4(\mathrm{e})$ ) and the internal one of $S_{R \mathrm{C} 2}$ width with lower contrast intensity (Figure 4(e)). The $R_{\mathrm{T}}$ ring (Figure 4(b)) consists of two sub-rings: the external one of $S_{R \mathrm{~T} 1}$ width with lower contrast intensity (Figure 4(f)) and the internal one of $S_{R \mathrm{~T} 2}$ width with higher contrast intensity (Figure 4(f)). The contrast inversion can be observed during a hypothetical move from the center of the rings $\mathrm{RC}$ and $\mathrm{RT}$ toward the outside: In Figure 4(a), the bright contrast ring is visible first and the dark one is visible first in Figure 4(b).

There is also a visible dark band $L_{1 \mathrm{C}}$ in the topogram of the SC surface of the C-type part (Figures 4(a), (c), and (e)). Bright band $L_{2 \mathrm{~T}}$ (Figures $4(\mathrm{~b}),(\mathrm{d})$, and (f)) may be observed in topograms obtained for T-type parts. This band appears after heat treatment. Fine parallel contrast bands (fine arrows, Figures 4(a), (c), and (e)), arranged along the $m$ direction, appear in the topograms of the C-type part. However, in the topograms of T-type parts, the bands mentioned above are not visible. The $m$ and $n$ directions from Figure 4(a) are parallel to the secondary dendrite arms and to the [100] and [010] crystallographic directions. The blurred, wider bands $\mathrm{H}$ visible in the topograms from Figures 4(b), (d), and (f) are parallel to the $h$ direction, which divides the angle between $m$ and $n$ in half. The $\mathrm{G}$ fragment (Figure 4(b)) with lack of contrast suggests that this area does not satisfy the Bragg's condition and fragments of contrast are shifted creating an overlapped high contrast $G_{\mathrm{T}}$ near the area $G$ of the topogram.

On the basis of the PALS spectra analysis, the defect concentration values were determined for both the A1 and A2 areas of as-cast and treated samples. The obtained values of defect concentration in the A1 and A2 areas (Figures 2(a) and 4(a)) of as-cast parts of the roots (C-type) and the defect concentration estimated for the analogous areas of the heat-treated parts (T-type) were averaged and summarized in Figure 5. For the purposes of analysis, it was assumed that the chemical composition of the undefected material (bulk) does not radically change qualitatively during heat treatment and changes occur in the distribution of matrix chemical components only, so the positron 


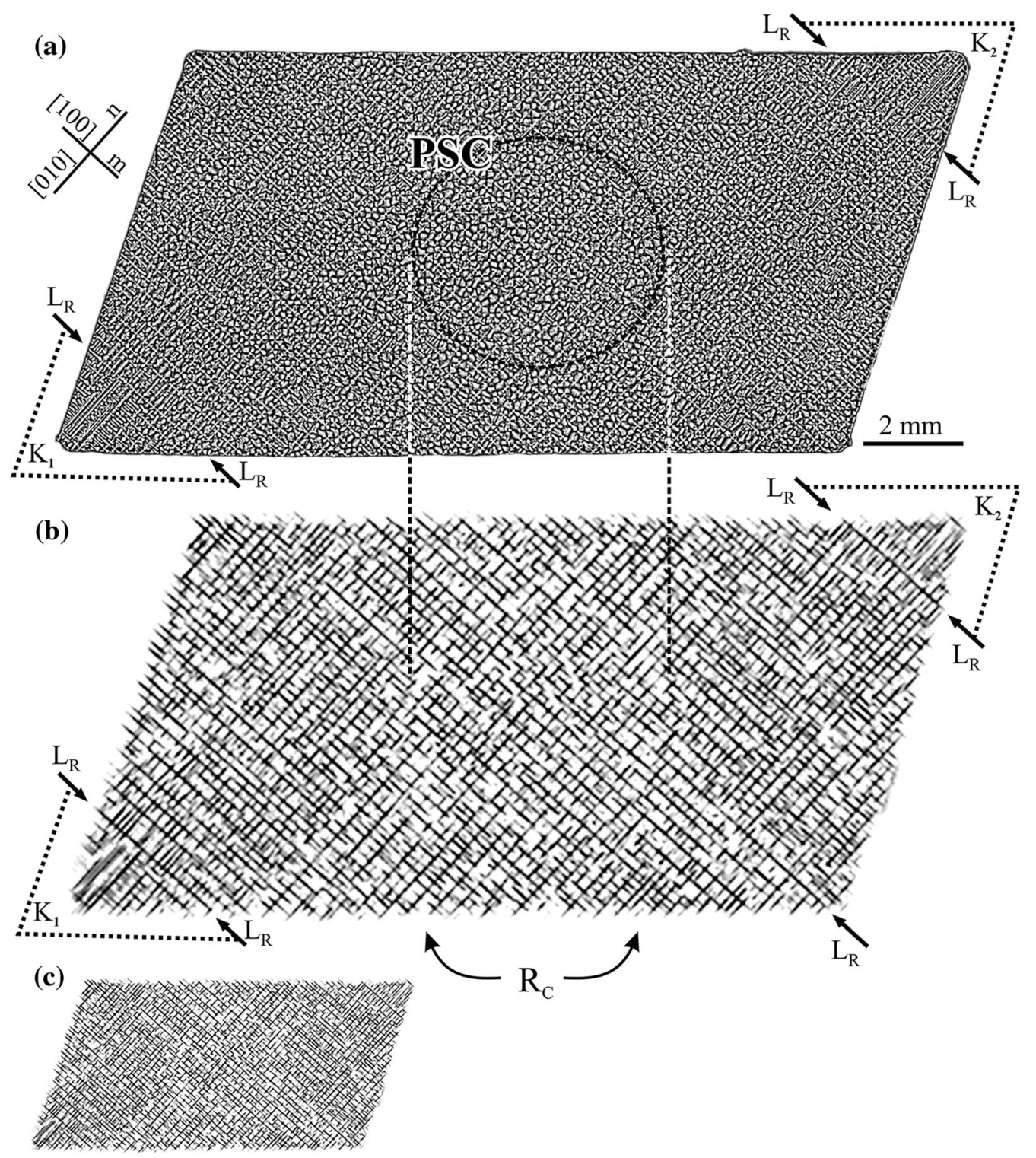

Fig. 3-(a) Typical macro-SEM image obtained for SC surface of C-type part, (b) dendritic structure presented in part (a) after computer processing, and (c) part (b) reduced in size.

annihilation lifetime in bulk is an invariant value, determined in the first step of analysis. The positron annihilation lifetime $\tau_{\mathrm{v}}$ was $134 \mathrm{ps}$, and this value is close to the literature values given for alloys based on $\mathrm{Ni}_{3} \mathrm{Al}$.

The results obtained by the PALS method suggest that in the as-cast parts of the root (C-type), there is such a high concentration of defects that it is not possible to indicate differences in the defect structure between two selected areas A1 and A2. The determined values of positron lifetime $(\tau=1.5 \mathrm{~ns})$, obtained as a result of a numerical analysis of the experimental spectrum, allow us to identify the type of dominant defects. The determined lifetime corresponds to volumetric defects of the micro-voids type also called the free volumes. ${ }^{[26]}$
Two types of defects were found in the T-type parts in both studied areas. Based on the positron lifetime calculations, the occurrences of mono-vacancies and dislocations were established. ${ }^{[17]}$ The defects concentration in the T-type parts (A1 and A2 areas) indicates that the dominant type of defects in both areas is vacancies. However, it should be noted that the concentration of each defect type in the discussed areas is different. It was observed that the vacancies concentration in the A1 area is about six times higher in relation to the dislocation concentration. The dislocation concentration in the A2 area is higher than in the A1 area, and additionally, the dislocation concentration in the A2 area is twice lower than the determined concentration of vacancies. The positron lifetimes for the vacancy in the area A1 $\left(\tau_{\mathrm{v}}=212 \mathrm{ps}\right)$ and in the area $\mathrm{A} 2\left(\tau_{\mathrm{v}}=220 \mathrm{ps}\right)$ were 


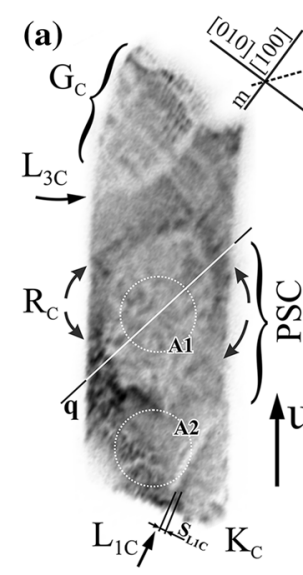

(c)

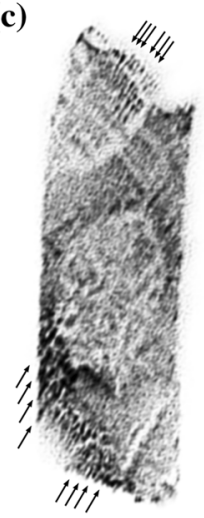

(e)<smiles>[TeH]</smiles>

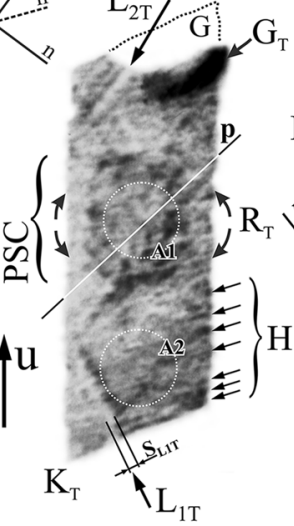

(d)

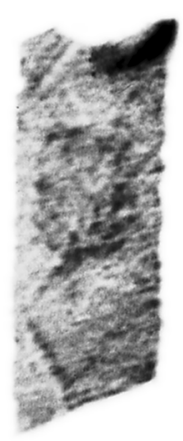

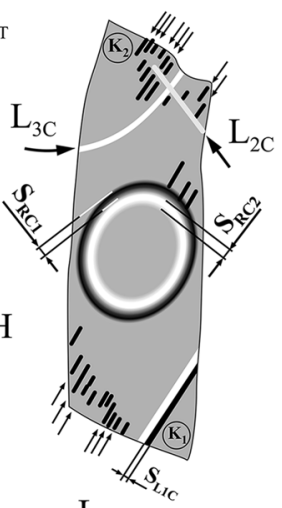

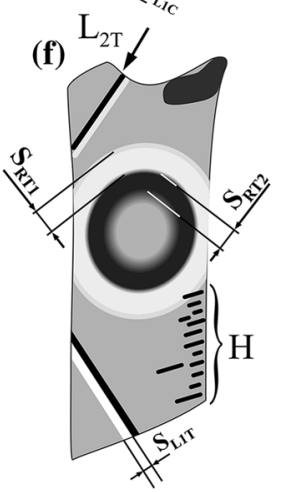

Fig. 4-Example of original topograms, obtained for (a) the SC surface of C-type part and $(b)$ the ST surface of T-type part; 113 reflection, $\mathrm{Cu} \mathrm{K} \alpha$ radiation; (c) and (d) the same topograms after computer processing; $(e)$ and $(f)$ schemes of contrast in topograms from parts (a) and (b).

determined. Similar calculations of positron lifetime were performed for dislocations in the area A1 $\left(\tau_{\mathrm{v}}=380 \mathrm{ps}\right)$ and in the area $\mathrm{A} 2\left(\tau_{\mathrm{v}}=410 \mathrm{ps}\right)$.

\section{DISCUSSION}

The difference in the dendrites' arrangement and arms' length inside and outside the PSC area (Figure 3) is related to the difference in growth kinetics. The growth of dendrites is not disturbed inside the PSC area on the $P_{\text {a }}$ plane (Figure 1(b)). Outside the PSC, the dendrite array in a whole root is conditioned by the rapid growth of the secondary dendrite arms on the $P_{\text {a }}$ plane (Figure 1(b)). ${ }^{[4,20]}$ The dendritic structure disorder in the $K_{1}$ and $K_{2}$ areas may be caused by local changes in the heat dissipation and by curvature of the solidification front in the narrow corner areas. ${ }^{[27]}$ It may also be the reason for the formation of contrast bands in topograms, separating the $\mathrm{K}_{1}$ and $\mathrm{K}_{2}$ areas (Figures 4(e), (c), and (a)), and this suggests a somewhat different crystal orientation of these areas in relation to other ones. Additionally, the secondary dendrite arms, growing in a transverse plane from the selector extension, do not reach into the $K_{1}$ and $K_{2}$ area, so the dendritic structure in these areas is different (Figures 3(a) and (b)) and often affected by the deflection process described in Reference 28. Structure alteration in the $R_{\mathrm{C}}$-ring region (Figure 3 ) similar to the specific shape of contrast of the PSC circle in topograms (Figures 4(a), (c), and (e)) is related to the circular shape of a selector continuer-root connection. This suggests that changes in the crystal orientation occur in this circle. The microstructure alteration visible on the

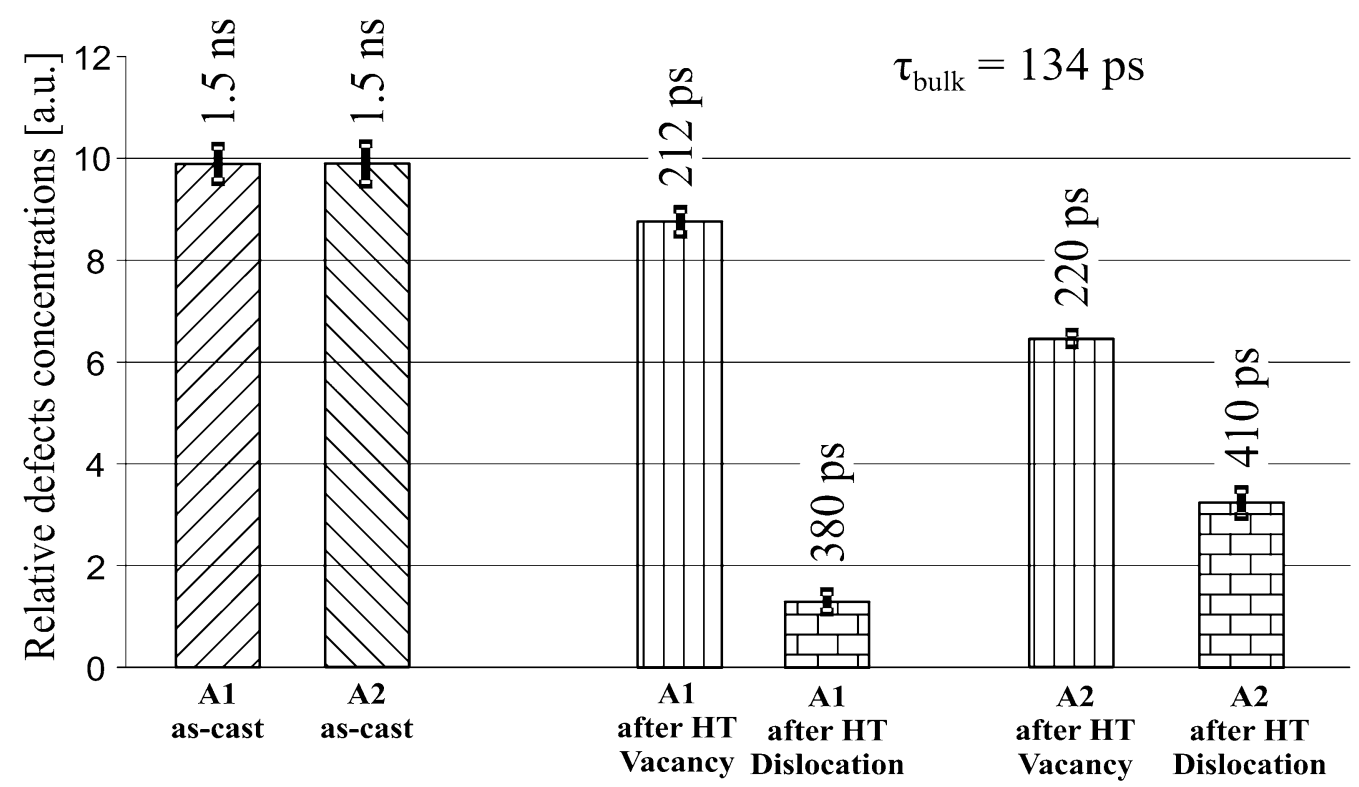

Fig. 5-Estimated defects concentrations with error bars and the determined values of positron lifetime in different types of defects in areas A1 and $\mathrm{A} 2$ of as-cast and treated parts of the root. 

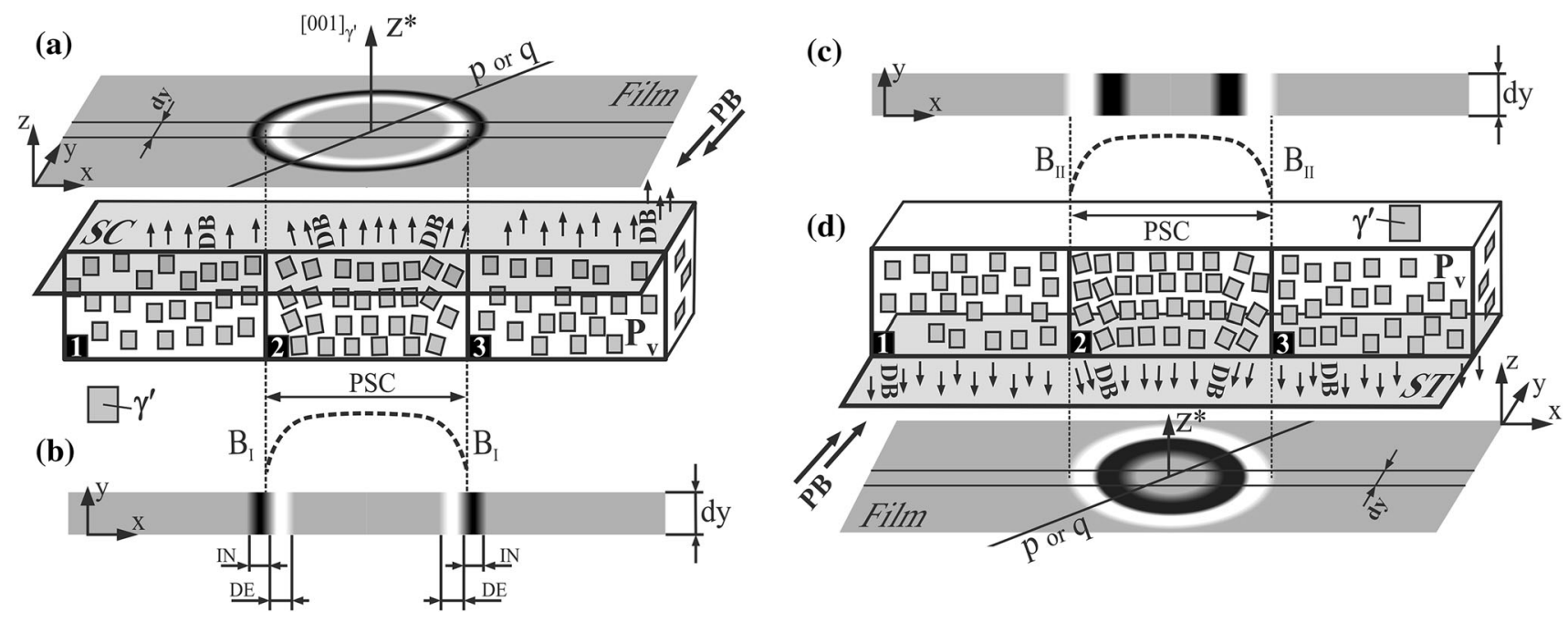

Fig. 6-(a) Scheme of contrast formation in the $R_{\mathrm{C}}$ ring of topograms (Figs. 4(a), (c), and (e)); (b) and (c) scheme of contrast in stripes $d_{y}$ for topogram obtained from SC and ST surface; and (d) scheme of contrast formation in the $R_{\mathrm{T}}$ ring of topograms (Figs. 4(b), (d), and (f)). Small arrows indicate $[001]_{\gamma^{\prime}}$ direction, $\mathrm{PB}, \mathrm{DB}$ - primary and diffracted X-ray beams, and the $\mathrm{B}_{\mathrm{I}}$ and $\mathrm{B}_{\mathrm{II}}$ bend is enlarged for figure clarity.

macro-SEM images (e.g., $K_{1}, K_{2}$ areas, $R_{\mathrm{C}}$ ring) and misoriented areas separated by boundaries similar to the LAB, called the "LAB-like" $\left(L_{1}, L_{2}, R\right.$-type rings) visible in topograms (Figure 4), suggest crystal misorientation. As a result of heat treatment, some defects disappear, e.g., the LAB-like, visualized in topogram as $L_{3 \mathrm{C}}$ (Figures 4(a), (c), and (e)) but some remain $\left(L_{1 \mathrm{C}}-L_{1 \mathrm{~T}}\right.$, Figures 4(a) and (b) and 4(c) and (d)). A new defects formation after heat treatment, e.g., the LAB-like, marked as $\mathrm{L}_{2 \mathrm{~T}}$ (Figures 4(b), (d), and (f)), was also observed.

For the treated parts of the roots, the positron lifetime for vacancy in the area $\mathrm{A} 1\left(\tau_{\mathrm{v}}=212 \mathrm{ps}\right)$ and in the area A2 $\left(\tau_{\mathrm{v}}=220 \mathrm{ps}\right)$ was determined. Similar calculations of positron lifetime were performed for dislocations in the area $\mathrm{A} 1\left(\tau_{\mathrm{v}}=380 \mathrm{ps}\right)$ and in the area $\mathrm{A} 2\left(\tau_{\mathrm{v}}=410\right.$ ps). Differences in the given positron lifetime values can be related to different chemical environments of the defects. The differences in the concentration of defects estimated by PALS in the area A1 and A2 of T-type parts are probably related to different kinetics of the crystallization process in these areas. Electron microscopy studies of a dendritic structure (Figure 3) indicate the differences in the dendritic array of the A1 area (included in the PSC) and the A2 area. It may be observed in Figure 3(b). Probably heat treatment implies changes in the structure of point and linear defects; however, areas with a relatively homogeneous distribution of dendrites related to undisturbed crystallization of the PSC area (contained the A1 area) are characterized by a different concentration of point and linear defects, related to the area (outside the PSC) where the crystallization process was disturbed for a moment by the step-change geometry of the mold (near the $P_{\text {a }}$ plane-Figure 1). The differentiated density of point defects in the studied areas may also be related to the inhomogeneity of the chemical composition in the defects environment, which suggests a different positron lifetime of the same-type defects. Thus, the treatment process does not homogenize the defect structure in the sub-microscopic scale. This requires further studies on a mechanism of $\gamma^{\prime}$ re-precipitating and its atomic ordering during heat treatment.

Elimination of inhomogeneity, created during dendritic crystallization of a complex-shape cast, is one of the aims of heat treatment. A transition of the crystallization front through the areas of the step-change geometry causes momentary changes in the crystallization kinetics due to the rapid growth of the secondary dendrite arms, perpendicular to the withdrawal direction. This growth takes place in a thin layer of the root (near $P_{\mathrm{a}}$-Figure 1) and occurs at a much higher growth rate than the withdrawal rate. As a result, a local disorder in crystal orientation and bending of dendrites, as well as the LAB-like formation, occur. ${ }^{[22]}$ The angle of misorientation of these boundaries can be estimated basing on the width of areas with increased or decreased contrast in the topogram. ${ }^{[13]}$ The areas of topograms of the increased contrast, derived from the SC surface, should be compared with the areas of the decreased contrast, derived from the ST surface, due to a contrast inversion resulting from the mirror-reflected SC and ST surfaces. In the case of the LAB $L_{1 \mathrm{C}}$ (Figures 4(a), (c), and (e)), the width $S_{L 1 c}$ is significantly smaller than the width $S_{\mathrm{L} 1 \mathrm{~T}}$ (Figures $4(\mathrm{~b}),(\mathrm{d})$, and (f)), which means that the angle of misorientation is significantly smaller. Similarly, as in a previous case, the width of the $S_{\mathrm{RC} 2}$ sub-ring is significantly smaller than the width of the $S_{\mathrm{RT} 2}$ sub-ring (Figures 4(e) and (f)). It follows that heat treatment caused an increase of the misorientation angle at the $R_{\mathrm{C}}$ and $L_{1 \mathrm{C}}$ boundaries. These macroscopic heterogeneities involve a defect generation at the microand nano-metric scale. Perfect heat treatment should, among others, eliminate dendritic segregation. Dissolution of the $\gamma^{\prime}$ phase after heat treatment and then its re-precipitation eliminates some defects. The basic physical process controlling homogenization is diffusion. Macroscopic inhomogeneity in the crystal 
orientation of the millimeters- or tenths of millimeters-sized areas cannot be eliminated by diffusion due to limited processing time. In addition, changes in the crystal orientation do not directly correlate with the concentration gradient of the components. Therefore, the defects related to the crystal misorientation may remain after treatment.

The effect visible in topograms as the $R_{\mathrm{C}}$ and $R_{\mathrm{T}}$ rings is related to the local crystal misorientation and can be explained on the basis of a scheme shown in Figure 6 and called the "flexure mirror-like effect." When the crystal orientation does not change throughout the analyzed areas (areas 1 and 3 of Figure 6(a)), the orientation contrast obtained in topograms is homogeneous and the intensity of diffracted beam is the same. In the case of the orientation changes in a certain area (area 2, Figure 6(a)), the diffracted beam overlaps neighboring areas increasing the intensity (area IN, Figure 6(b)); meanwhile, in the expected place, there is a decreased contrast (area DE, Figure 6(b)). Two types of contrast shifts can be created in topograms, outside (Figures 6(a) and (b)) and inside (Figures 6(c) and (d)), in relation to the PSC area, and two types of misorientation can be determined from topograms: the convex $\left(B_{\mathrm{I}}-B_{\mathrm{I}}\right.$, Figure $\left.6(\mathrm{~b})\right)$ and the concave $\left(B_{\mathrm{II}}-B_{\mathrm{II}}\right.$, Figure 6(c)) mirror-like effect, respectively. The $B_{\mathrm{I}}-B_{\mathrm{I}}$ and $B_{\mathrm{II}}-B_{\mathrm{II}}$ curves describe a distribution of $[001]_{\gamma^{\prime}}$ crystallographic direction, which is perpendicular to these curves along the $X$ axis in the PSC region. This is extremely important when blades are obtained by seeding. ${ }^{[29]}$ It was assumed that topograms are created only by diffraction from the $\gamma^{\prime}$ phase (volume content about 70 pct). The example shown in Figure 6 occurs for a symmetrical deviation from the growth direction $Z^{*}$, but in other cases, the contrast rings are not centrosymmetric. The presented mirror-like effect may be related to the crystallization front bend in a selector continuer or in a seed.

A high concentration of volumetric defects revealed by PALS method in the as-cast parts of the roots may be related to a porosity. ${ }^{[30]}$ Nucleation of pores depending on the local stress level in the interdendritic melt may be driven by stress relaxation after pore nucleation ${ }^{[31]}$ that appeared near areas of step-changes in the mold geometry. The signal of positron annihilation from volumetric defects is high and prevents detecting vacancies and dislocations in an as-cast state. After heat treatment, the volumetric defects disappear and the vacancies with the dislocations may be detected by PALS.

\section{CONCLUSION}

The work carried out in this article allows the following specific conclusions to be drawn:

1. Heat treatment does not eliminate most of the macroscopic defects that are associated with misorientation of dendrites. The misorientation angle of boundaries similar to low-angle boundaries (like-LAB) increases after heat treatment.
2. In the region near the rim of the selector continuer projection (PSC), a specific macroscopic distribution of crystal misorientation is present in both as-cast and heat-treated roots. This distribution has the character of a flexure mirror-like effect and does not disappear after heat treatment.

3. The as-cast regions of the blade roots, located near the selector continuer, contain high concentrations of micro-voids and pores; these make it impossible to determine by PALS the differences in vacancies and dislocations concentration.

4. It has been found by PALS that for heat-treated parts of the roots, there are differences in the concentration and type of defects between the area of PSC and beyond it. Inside it the concentration of dislocations is found to be lower than outside it. The concentration of vacancies inside the PSC area is higher.

5. Additionally, it was found that both inside and outside the PSC area after treatment, the concentration of vacancies is higher than the concentration of dislocations. This may be caused by migration of dislocations during treatment to the like-LAB and their annihilation. This assumption is consistent with the fact that the LAB-like angle of misorientation increases after heat treatment.

6. In this article, it has been demonstrated that the combination of $\mathrm{X}$-ray topography and positron annihilation spectroscopy allows for a multiscale structural examination for the determination of defect structure, including the low-angle boundary (LAB) areas and nano- and micro-scale defects.

\section{OPEN ACCESS}

This article is distributed under the terms of the Creative Commons Attribution 4.0 International License (http://creativecommons.org/licenses/by/4.0/), which permits unrestricted use, distribution, and reproduction in any medium, provided you give appropriate credit to the original author(s) and the source, provide a link to the Creative Commons license, and indicate if changes were made.

\section{REFERENCES}

1. R.C. Reed: The Superalloys: Fundamentals and Applications, Cambridge University Press, Cambridge, UK, 2006.

2. M.J. Donachie and S.J. Donachie: Superalloys-A Technical Guide, 2nd ed., ASM International, Materials Park, 2002.

3. T.M. Pollock: J. Propul. Power, 2006, vol. 22 (2), pp. 361-74.

4. G.E. Fuchs: Mater. Sci. Eng. A, 2001, vol. 300, pp. 52-60.

5. J.R. Li, J.Q. Zhao, S.Z. Liu, and M. Han: Superalloys 2008, TMS, 2008, pp. 443-51.

6. N. Stanford, A. Djakovic, B. Shollock, M. McLean, N. D'Souza, and P. Jennings: Superalloys 2004, TMS, 2004, pp. 719-26.

7. N. Stanford, A. Djakovic, B.A. Shollock, M. McLean, N. D'Souza, and P.A. Jennings: Scr. Mater., 2004, vol. 50, pp. 159-63. 
8. V.A. Vorontsov, L. Kovarik, M.J. Mills, and C.M.F. Rae: Acta Mater., 2012, vol. 60, pp. 4866-78.

9. M. Huang, Z. Cheng, J. Xiong, J. Li, J. Huc, Z. Liu, and J. Zhu: Acta Mater., 2014, vol. 76, pp. 294-305.

10. E. Rzyankina, D. Szeliga, N. Mahomed, and A. Nowotnik: Appl. Mech. Mater., 2013, vol. 372, pp. 54-61.

11. M. Ramsperger, L. Roncery, I. Lopez-Galilea, R.F. Singer, W. Theisen, and C. Körner: Adv. Eng. Mater., 2015, vol. 17 (10), pp. 1486-93.

12. P. Zhang, Q. Zhu, G. Chen, H. Qin, and Ch. Wang: Materials, 2015, vol. 8, pp. 6179-94.

13. W. Bogdanowicz: Scr. Mater., 1997, vol. 37 (6), pp. 829-35.

14. K.G. Lipetzky, R.E. Green, Jr., and P.J. Zombo: Nondestructive Characterization of Materials VIII, Springer, Boston, 1998, pp. $423-30$.

15. A. Onyszko, W. Bogdanowicz, K. Kubiak, and J. Sieniawski: Cryst. Res. Technol., 2010, vol. 45 (12), pp. 1326-32.

16. J. Krawczyk, W. Bogdanowicz, J. Sieniawski, and K. Kubiak: Acta Phys. Pol. A, 2016, vol. 130 (4), pp. 1100-03.

17. H.E. Schaefer, F. Baier, M.A. Müller, K.J. Reichle, K. Reimann, A.A. Rempel, K. Sato, F. Ye, X. Zhang, and W. Sprenge: Phys. Status Solidi B, 2011, vol. 248 (10), pp. 2290-99.

18. S. van Petegem, J. Kuriplach, M. Hou, E.E. Zhurkin, D. Segers, A.L. Morales, S. Ettaoussi, C. Dauwe, and W. Mondelaers: Mater. Sci. Forum, 2001, vols. 363-65, pp. 210-12.

19. J.K. Tien, S.J. Tao, J.P. Wallace, and S. Purushothaman: Electron and Positron Spectroscopies in Materials Science and Engineering, Academic Press, New York, 1979, pp. 73-118.
20. T. Sadowski and P. Golewski: Loadings in Thermal Barrier Coatings of Jet Engine Turbine Blades: An Experimental Research and Numerical Modeling, Springer, Singapore, 2016.

21. J. Krawczyk, A. Tondos, W. Bogdanowicz, and R. Paszkowski: Powder Metall. Met. Ceram., 2017, vol. 56 (7-8), pp. 481-86.

22. W. Bogdanowicz, A. Tondos, J. Krawczyk, R. Albrecht, and J. Sieniawski: Acta Phys. Pol. A, 2016, vol. 130 (4), pp. 1107-09.

23. J. Krawczyk, W. Bogdanowicz, and T. Goryczka: Cryst. Res. Technol., 2010, vol. 45 (12), pp. 1321-25.

24. J. Kansy: Nucl. Instrum. Methods Phys. Res. Sect. A, 1996, vol. 374 , pp. $235-44$.

25. J.E. Miller, M. Strangwood, S. Steinbach, and N. Warnken: Proceedings of the 5th Decennial International Conference on Solidification Processing, Old Windsor, July 2017.

26. O. Melikhova, J. Kuriplach, I. Prochazka, J. Cizek, M. Hou, E. Zhurkin, and S. Pisov: Appl. Surf. Sci., 2008, vol. 255, pp. 157-59.

27. D. Szeliga, K. Kubiak, and J. Sieniawski: J. Mater. Process. Technol., 2016, vol. 234, pp. 18-26.

28. W. Bogdanowicz, J. Krawczyk, A. Tondos, and J. Sieniawski: Cryst. Res. Technol., 2017, vol. 52, p. 1600372.

29. N. D'Souza, P.A. Jennings, X.L. Yang, H.B. Dong, P.D. Lee, and M. McLean: Metall. Mater. Trans. B, 2005, vol. 36B, pp. 657-65.

30. K. Kubiak, D. Szeliga, J. Sieniawski, and A. Onyszko: Handbook of Crystal Growth: Bulk Crystal Growth, 2nd ed., Elsevier, Amsterdam, 2015, pp. 413-57.

31. A.J. Torroba, O. Koeser, L. Calba, L. Maestro, E. Carreño-Morelli, R. Mehdi, S. Milenkovic, I. Sabirov, and J. Lorca: Integr. Mater. Manuf. Innov., 2014, vol. 3 (1), pp. 1-25. 